\title{
Impaired Retinal Circulation during Axitinib Treatment for Metastatic Renal Cell Carcinoma
}

\author{
Masashi Kimura $^{a}$ Sentaro Kusuhara ${ }^{a}$ Mizuki Tagami ${ }^{b}$ \\ Makoto Nakamura ${ }^{a}$ \\ aDivision of Ophthalmology, Department of Surgery, Kobe University Graduate School of \\ Medicine, Kobe, Japan; 'bepartment of Ophthalmology, Kobe Kaisei Hospital, Kobe, Japan
}

\section{Keywords}

Adverse event · Axitinib · Renal cell carcinoma - Retinal circulation · Vascular endothelial growth factor

\begin{abstract}
Purpose: Axitinib, an orally administered vascular endothelial growth factor receptors 1, 2, and 3 inhibitor, is widely used as the second-line treatment for metastatic renal cell carcinoma. We present a case of metastatic renal cell carcinoma who developed a novel ocular adverse event, impaired retinal circulation, during axitinib therapy. Methods: This is an observational case report. Results: A 57-year-old male who had been treated with axitinib for metastatic renal cell carcinoma for 2 years presented in August 2015. He complained of sudden-onset abnormal visual field in his right eye. His right eye exhibited multiple soft exudates on fundus photography and a significant fluorescein filling delay of the retinal vessels on fluorescein angiography. His best corrected visual acuity (BCVA) was 20/20 in the right eye, and a cecocentral scotoma was detected by Goldmann perimeter. As axitinib could have been responsible for impaired retinal circulation, axitinib was terminated and switched to temsirolimus. The soft exudates gradually subsided and the patients' symptoms got better, but his right BCVA dropped to 20/63 3 months after the end of axitinib treatment with worsening of his general condition. Conclusion: Clinicians should be aware of retinal circulatory disorder that can occur in patients under axitinib treatment.




\section{Introduction}

Since solid tumours require oxygen and nutrients for growth and metastasis, sprouting angiogenesis is induced from the surrounding vessels to satisfy the demand and is called tumour angiogenesis. The vascular endothelial growth factor (VEGF) signalling pathway plays a pivotal role in tumour angiogenesis, and it is proved that antiangiogenic drugs targeting the VEGF signalling pathway produce beneficial effects in cancer treatment. Axitinib is an orally administered drug that potently and selectively inhibits VEGF receptors 1, 2, and 3 [1]. Based on the data from the Phase 3 AXIS Trial, the US Food and Drug Administration has approved axitinib in 2012 for the treatment of metastatic renal cell carcinoma that has progressed despite first-line therapy [2]. Adverse events with the use of axitinib such as diarrhoea, hypertension, and fatigue are considered tolerable [2, 3], but it remains unknown whether axitinib may affect retinal circulation or not. Here, we present a case of impaired retinal circulation who had been treated with axitinib for metastatic renal cell carcinoma.

\section{Case Presentation}

A 57-year-old male with a history of hypertension, hypothyroidism, and hyperuricemia was diagnosed as having renal cell carcinoma with adrenal metastasis. He underwent right nephrectomy and bilateral partial adrenal resection in July 2012 followed by adjuvant chemotherapy with sunitinib (50 mg/day). In May 2013, the treatment regimen was changed from sunitinib to axitinib ( $10 \mathrm{mg} /$ day) as computed tomography revealed the presence of recurrent or metastatic tumours in the lung, adrenal gland, thigh adductor muscle, and liver. The patient also received CyberKnife radiosurgery for brain metastasis and then transarterial embolization for metastatic liver tumours in October 2013, 5 months after the commencement of axitinib treatment. In July 2015, 2 years after the initiation of axitinib treatment, the patient noticed a sudden-onset abnormal visual filed in his right eye and was referred to the Department of Ophthalmology, Kobe Kaisei Hospital. His best corrected visual acuity (BCVA) was $20 / 20$ in the right eye and 20/12 in the left eye. His right fundus showed multiple soft exudates while his left fundus exhibited normal appearance (Fig. 1a, b). Fluorescein angiography disclosed a significant filling delay of fluorescein dye in the right eye, and indocyanine green angiography revealed no remarkable abnormalities except for blocked hypofluorescence (Fig. 2). In August 2015, 2 weeks after the initial presentation to Kobe Kaisei Hospital, the patient was referred to the Ophthalmology Department of Kobe University Hospital for further examination and treatment as the symptom remained unchanged. On examination, his BCVA was 20/20 in the right eye and 20/12 in the left eye, and intraocular pressure was $12 \mathrm{~mm} \mathrm{Hg}$ in the right eye and $14 \mathrm{~mm} \mathrm{Hg}$ in the left eye. Goldmann perimeter detected a cecocentral scotoma in the right eye, but there was no relative afferent pupillary defect. Slit-lamp biomicroscopy revealed no abnormality in the anterior segment of either eye. Fundus findings were the same as those in Kaisei Hospital. The laboratory data showed white blood cell count 6,500/ $\mu \mathrm{L}$, haemoglobin $19.3 \mathrm{~g} / \mathrm{dL}$, haematocrit $60.5 \%$, platelet count $293,000 / \mu \mathrm{L}$, C-reactive protein $2.85 \mathrm{mg} / \mathrm{dL}$, creatinine $1.58 \mathrm{mg} / \mathrm{dL}$, total cholesterol $332 \mathrm{mg} / \mathrm{dL}$, triglycerides $255 \mathrm{mg} / \mathrm{dL}$, and glucose $103 \mathrm{mg} / \mathrm{dL}$. Systemic conditions that might have caused the ocular abnormality, such as carotid artery occlusion and collagen disease, were not identified after inquiry with the attending doctor. As axitinib could have been responsible for impaired retinal circulation, axitinib was terminated and switched to temsirolimus (mTOR inhibitor) by the discretion of the urologist in charge. The soft exudates then gradually subsided by 2 months 
(Fig. 1c, d) although his right BCVA dropped to 20/63. Further detailed examinations were unable to be made because his general condition worsened due to progression of brain metastasis approximately 3 months after the end of axitinib treatment.

\section{Discussion}

We experienced a case of retinal circulatory disorder during axitinib treatment. Axitinib offers improved progression-free survival in the second-line treatment setting for metastatic renal cell carcinoma [2], and therefore it has been used worldwide. However, it is theoretically probable that axitinib increases the risk of arterial thromboembolic events because axitinib potently inhibits VEGF signalling (50-450 times greater than first-generation VEGF receptor inhibitors) [2] and anti-VEGF agents are known to increase the risk of arterial thromboembolic events [4]. In fact, there is a case report suggesting that axitinib treatment may have been associated with acute myocardial infarction [5]. In our case, no obvious embolus was detected by funduscopy, but the findings of fluorescein angiography and the clinical course strongly suggest the presence of a transient impeded blood flow in the central retinal artery which might be associated with a long-term use of a potent VEGF signalling inhibitor. We speculate that axitinib long damaged retinal vessels as well as large vessels which nourish the eye balls and caused the retinal circulatory event at the time when cumulated damage exceeded a certain threshold. As this situation would not always simultaneously take place in both eyes, we think it is possible that the ipsilateral retinal manifestation was attributed to axitinib use. CyberKnife radiosurgery or transarterial embolization may have caused the impaired retinal circulation in our case, but both retinas had not been intensively irradiated and transarterial embolization had been performed approximately 2 years before. Therefore, these treatments are unlikely to be associated with retinal circulatory impairment. It is well known that mTOR inhibitor has an anti-VEGF effect. However, mTOR inhibitor only inhibits PI3K cascade, while axitinib hinders both PI3K and Ras pathways, meaning that axitinib is theoretically more potent than mTOR inhibitor in terms of inhibition of the VEGF/VEGF receptor pathway. We guess that switching to mTOR inhibitor contributed to improved retinal circulation via increased serum VEGF level, although the serum VEGF level was not monitored in our case. We admit that the reason for declined visual acuity after the discontinuation of axitinib treatment in our case is unclear because the decreased level of consciousness due to brain metastasis precluded further examinations. The point of this case report is not to clarify whether axitinib truly caused this ocular abnormality, but to convey the fact that a considerable retinal circulatory disorder occurred in a patient under axitinib treatment. In conclusion, clinicians should be aware of impaired retinal circulation which potentially leads to visual impairment in patients under axitinib treatment.

\section{Statement of Ethics}

This study complied with the tenets of the Declaration of Helsinki. Approval by the IRB was exempted as this was a single case report. Written informed consent was obtained from the patient for publication of this case report and any accompanying images. 


\section{Disclosure Statement}

The authors indicate no financial support and no proprietary or conflict of interest in this case report.

\section{Author Contributions}

M. Kimura, S. Kusuhara, M. Tagami: data collection. M. Kimura, S. Kusuhara: manuscript preparation. M. Nakamura: supervision. All authors read and approved the final manuscript.

\section{References}

1 Hu-Lowe DD, Zou HY, Grazzini ML, Hallin ME, Wickman GR, Amundson K, et al. Nonclinical antiangiogenesis and antitumor activities of axitinib (AG-013736), an oral, potent, and selective inhibitor of vascular endothelial growth factor receptor tyrosine kinases 1, 2, 3. Clin Cancer Res. 2008 Nov;14(22):7272-83.

2 Rini BI, Escudier B, Tomczak P, Kaprin A, Szczylik C, Hutson TE, et al. Comparative effectiveness of axitinib versus sorafenib in advanced renal cell carcinoma (AXIS): a randomised phase 3 trial. Lancet. 2011 Dec;378(9807):1931-9.

3 Wang H, Man L, Li G, Huang G, Wang J. Comparative efficacy and safety of axitinib versus sorafenib in metastatic renal cell carcinoma: a systematic review and meta-analysis. OncoTargets Ther. 2016 Jun;9: 3423-32.

4 Scappaticci FA, Skillings JR, Holden SN, Gerber HP, Miller K, Kabbinavar F, et al. Arterial thromboembolic events in patients with metastatic carcinoma treated with chemotherapy and bevacizumab. J Natl Cancer Inst. 2007 Aug;99(16):1232-9.

5 Gürel E, Günaydın ZY, Karaoğlanoğlu M, Kırıș T. Acute myocardial infarction induced by axitinib. Anadolu Kardiyol Derg. 2014 Nov;14(7):661. 


\section{Case Reports in Ophthalmology}

www.karger.com/cop

Kimura et al.: Axitinib and Impaired Retinal Circulation
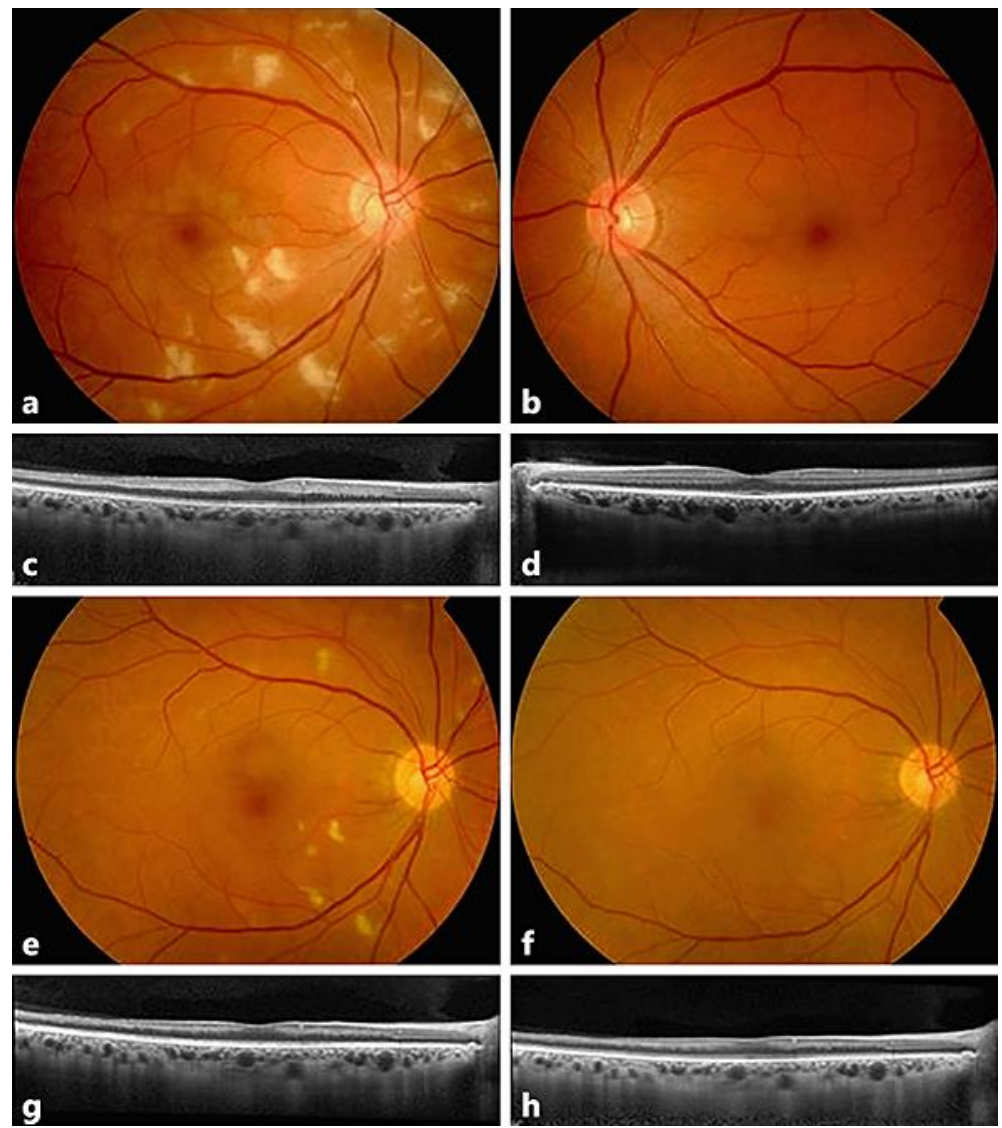

Fig. 1. Colour fundus photographs (taken by TRC-50DX, Topcon, Tokyo, Japan) and horizontal OCT images (acquired by Heidelberg Spectralis, Heidelberg Engineering, Heidelberg, Germany) before and after the cessation of axitinib treatment. a, b Fundus images in July 2015. Multiple large soft exudates were seen in the right eye while no abnormality was observed in the left eye. c, $\mathbf{d}$ OCT showed a hyperreflective inner layer in the right eye and a normal appearance in the left eye. Retinal thickness at the temporal macula appeared thinner in the right eye than in the left eye. e The soft exudates decreased in size and number within 2 weeks after stopping axitinib intake. $\mathbf{g}$ The hyperreflective inner layer became inconspicuous on OCT. $\mathbf{f}, \mathbf{h}$ The right eye returned to normal appearance 2 months after the end of axitinib treatment. 


\section{Case Reports in Ophthalmology}
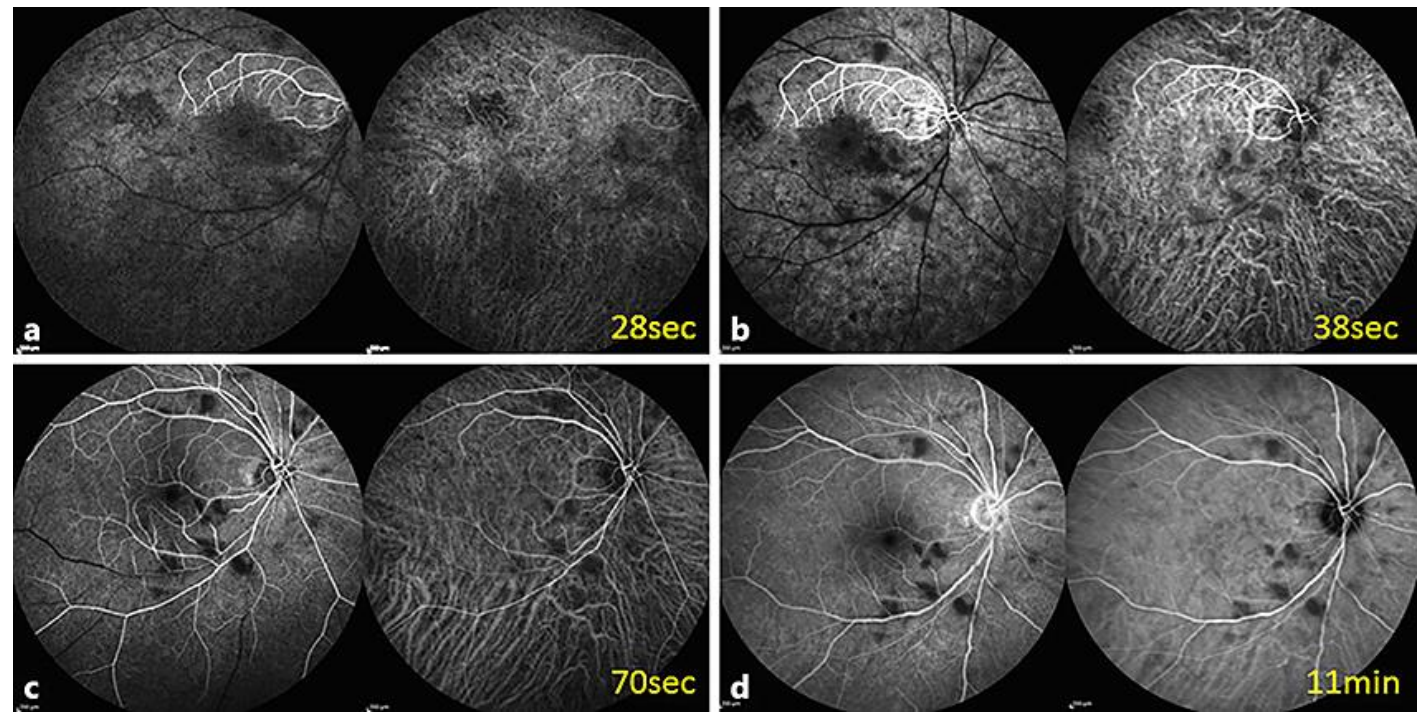

Fig. 2. Fluorescein angiogram/indocyanine green angiogram (FA/IA) of the right eye in July 2015. a The choroidal flush and the following arterial fluorescence became visible $28 \mathrm{~s}$ after injection on FA. b Within the next $10 \mathrm{~s}$, fluorescein perfusion was observed only in the superotemporal branch of the central retinal artery on FA. Choroidal filling was normal on IA. c The laminar pattern of retinal venous flow was still detectable on FA $70 \mathrm{~s}$ after injection. $\mathbf{d}$ There was no evident abnormal finding except for fluorescein blocking corresponding to soft exudates on the late phase. FA/IA was performed with HRA (Heidelberg Engineering). 